\title{
Earth's rotation variability triggers explosive eruptions in subduction zones
}

\author{
Gianluca Sottili ${ }^{1 *}$ (D), Danilo M. Palladino ${ }^{2}$, Marco Cuffaro ${ }^{3}$ and Carlo Doglioni ${ }^{2,3}$
}

\begin{abstract}
The uneven Earth's spinning has been reported to affect geological processes, i.e. tectonism, seismicity and volcanism, on a planetary scale. Here, we show that changes of the length of day (LOD) influence eruptive activity at subduction margins. Statistical analysis indicates that eruptions with volcanic explosivity index (VEI) $\geq 3$ alternate along oppositely directed subduction zones as a function of whether the LOD increases or decreases. In particular, eruptions in volcanic arcs along contractional subduction zones, which are mostly E- or NE-directed, occur when LOD increases, whereas they are more frequent when LOD decreases along the opposite W- or SW-directed subduction zones that are rather characterized by upper plate extension and back-arc spreading. We find that the LOD variability determines a modulation of the horizontal shear stresses acting on the crust up to $0.4 \mathrm{MPa}$. An increase of the horizontal maximum stress in compressive regimes during LOD increment may favour the rupture of the magma feeder system wall rocks. Similarly, a decrease of the minimum horizontal stress in extensional settings during LOD lowering generates a larger differential stress, which may enhance failure of the magma-confining rocks. This asymmetric behaviour of magmatism sheds new light on the role of astronomical forces in the dynamics of the solid Earth.
\end{abstract}

\section{Introduction}

The angular velocity of Earth's rotation varies in response to astronomical forcing and terrestrial dynamics. Tidal drag due to the lunisolar gravitational torque results into decadal to secular variations of the length of day (LOD) (Lambeck 1980; Riguzzi et al. 2010; Ide and Tanaka 2014; Palladino and Sottili 2014). Also, shortterm (fortnightly to annual) LOD fluctuations occur due to zonal tides (Jault et al. 1988; Kane and Trivedi 1990) whilst daily to multi-year LOD changes are induced by angular momentum exchanges between solid Earth, hydrosphere and atmosphere (Barnes et al. 1983; Marcus et al. 1998; Le Mouël et al. 2010; Palladino and Sottili 2014; Sottili 2014), Earth core-mantle coupling (Mound and Buffett 2003) and other internal forces (i.e. earthquakes and post-glacial rebound; Chao et al. 2014). Hence, the LOD temporal pattern is the result of the superposition of periodic, quasi-periodic and stochastic oscillations on different timescales. Here, we focus on annual Earth's spin changes, which are dominantly induced by atmospheric

\footnotetext{
* Correspondence: gianluca.sottili@uniroma1.it

${ }^{1}$ Istituto di Geologia Ambientale e Geoingegneria (IGAG) —CNR, 00015

Monterotondo, Rome, Italy

Full list of author information is available at the end of the article
}

angular momentum (AAM) exchanges between the atmosphere and solid Earth (Rosen and Salstein 1985). Earth's spin perturbations release substantial amounts of gravitational energy (Press and Briggs 1975), with effects on the crustal deformation rate and mass redistribution on the plate tectonic scale (Riguzzi et al. 2010; Wang et al. 2000; Milyukov et al. 2013) and the time-space distribution of large earthquakes (Anderson 1974; Varga et al. 2005). Thus, Earth's rotation can actually contribute to the state of stress accumulated in the different Earth's discontinuities, e.g. lithospheric plate boundaries and lithosphereasthenosphere boundary (Doglioni et al. 2011).

Volcanic activity is strongly affected by crustal stress changes on different time and space scales (e.g. due to global tectonism, glacio-eustatism and tidal forces; Kutterolf et al. 2012; Sottili et al. 2007). The recently described causal relationship between LOD changes and volcanic eruptions (Palladino and Sottili 2014) provides the background to understand how yearly LOD changes perturb the state of stress in specific geodynamic contexts.
焦 Springer

(c) 2015 Sottili et al. Open Access This article is distributed under the terms of the Creative Commons Attribution 4.0 International License (http://creativecommons.org/licenses/by/4.0/), which permits unrestricted use, distribution, and reproduction in any medium, provided you give appropriate credit to the original author(s) and the source, provide a link to the Creative Commons license, and indicate if changes were made. 


\section{Data set and analyses}

We investigate the effects of Earth rotational changes on volcanism along convergent boundaries. From a database including 620 explosive eruptions with volcanic explosivity index (VEI) $\geq 3$ since AD 1850 to present (Palladino and Sottili 2014; Siebert and Simkin 2002) based on the Smithsonian Institution catalogue, Global Volcanism Program, 2015, available online at www.volcano.si.edu, we analyse the occurrences of 422 events at subductionrelated volcanoes with respect to LOD time series (International Earth Rotation and Reference System Service, Observatoire de Paris, IERS EOP PC 2015, available online at http://hpiers.obspm.fr/eop-pc/; Bizouard and Gambis 2009). The VEI, ranging on a scale from 0 to 8 , is an empirical measure based on magnitude (erupted volume) and intensity (column height) of explosive eruptions. The VEI $\geq 3$ threshold for the study data set was chosen to minimize the uncertainty in the reported occurrences of minor eruptions back in time. A VEI $\geq 3$ volcanic event typically erupts at least $0.01 \mathrm{~km}^{3}$ of tephra, with a column height in excess of $5 \mathrm{~km}$. For each volcano, we considered only the highest VEI eruption in case of multiple events per year (see Additional file 1). The database provides quite reliable years of occurrence of volcanic events, but it is uncertain about the exact dates (day, month) of eruption onset, especially in remote regions. For this reason, and due to the resolution of the database, we considered annual LOD changes $b e$ fore the year of occurrence of volcanic eruptions to make sure that LOD changes actually preceded a specific eruptive event.

Of the 422 selected events, 166 are located along E- or NE-directed and 256 along W- or SW-directed subduction zones, i.e. following or opposing the mantle flow inferred from the net rotation of the lithosphere relative to the underlying mantle (Crespi et al. 2007; Cuffaro and Doglioni 2007 and references therein). Due to the irregular morphology of plate boundaries and the undulated mainstream of plate motions, subduction processes can occur with normal or oblique motion directions of sinking slabs, relative to the global mainstream of plates that is "westerly" polarized. Therefore, practically any direction of subduction strike can be observed. However, subduction zones can be subdivided into two main classes along the mainstream of plates that has a tectonic equator at about $30^{\circ}$ relative to the geographic equator, being the two classes subdivided as a function if the subduction is in favour or against the relative mantle flow. Apart from very few exceptions that can indicate the initiation of a subduction flip (e.g. Northern Japan), the two end members are generally characterized by the subduction hinge converging relative to the upper plate (E- or NE-directed slabs), whereas it diverges along the opposite subduction zones (W- or SW-directed slabs), determining, respectively, extension and contraction in the upper plate (Doglioni et al. 2007). However, it must be pointed out that the distinction among the two types of subduction zones is then not E-W but along the westerly polarized undulate flow of plate motion (Cuffaro and Doglioni 2007; Doglioni and Panza 2015), which is for example W-directed in the western Pacific, Caribbean, Scotia and Banda arc slabs and the E-directed slab in the Americas cordilleras and becoming NE- to NNE-directed along the Makran, Himalayas and Indonesia subduction zones, mimicking and paralleling the similar trend of the spreading direction of the Earth's three main oceanic ridges (East Pacific Rise, Mid-Atlantic Ridge and Indian Ridge). Despite that the main directions of plate motions are not E-W, in order to simplify our analysis, E- and Wdirected subduction zones are here categorized, investigating their trench mean azimuth, at the Earth's surface. We consider the slab dip directions worldwide, from the Slab 1.0 database (Hayes et al. 2012), and we computed the mean azimuth of the normal lines to the trench segments. From this database, we select the geometry of the trenches. We do not account for the quality of the database. Some regions are better described, whereas others are poorly described. Our purpose is not to obtain equally spaced trench segments. We consider couples of consecutive points of each trench line, and then, we compute the azimuth of the normal line to the segment connecting each couple. We sampled the obtained normal line azimuths to have clear representation of the data, compared with the other reported data, such as volcano locations and plate velocities obtained by Gripp and Gordon (2002) (see Additional file 1). We applied to each subduction zone a proper sampling procedure, compared with the dimensions of the investigated area, and the length of the trench line.

The trenches are subdivided following the shape of the tectonic equator, described by Crespi et al. (2007) or Cuffaro and Doglioni (2007). The shape of the tectonic equator, the lithosphere net rotation main directions and velocities are reported in Additional file 1: Figure S1. As an example, the Sumatra-Java-Banda system, in which trajectories of the net rotation of the lithosphere produce undulations in that region, is characterized by a W-directed subduction zone at Banda arc and a NEdirected one at Sumatra.

Some arc geometry analysed in this paper, i.e. the Caribbean and Banda arcs, or other trench segments where active volcanoes are located are not in the database from Hayes et al. (2012). In these cases, we used plate boundary data from DeMets et al. (2010), or Gridded bathymetry data (e.g. a global 30-arc sec-interval grid from GEBCO http://www.gebco.net/data_and_products/gridded_bathymetry_data/) to define the location of 
the trenches. The choice of the trench length also takes into account the locations of the active volcanoes analysed. Additional trench data are represented as dashed lines, whereas trenches from Hayes et al. (2012) are reported as solid lines (see Additional file 1).

Classification of E- and W-directed subduction zones is made only considering the resulting mean azimuths. When these data show an E- or W-component, the subduction geometry is defined as E- or W-directed, respectively (see Additional file 1). Basically, this simplified criterion gives results comparable with those obtained by Crespi et al. (2007) and Cuffaro and Doglioni (2007), concerning the E- and W-directed categories. As an example, the NE- or NNE-directed Indonesian subduction zone falls into the "E" class. To define Earth's spin changes, we consider $\triangle \mathrm{LOD}$ as the difference between astronomically measured universal time (UT1) and international atomic time (French, Temps Atomique International; TAI):

$$
\Delta \mathrm{LOD}=-\mathrm{LOD}_{0} \frac{d(\mathrm{UT} 1-\mathrm{TAI})}{d(\mathrm{TAI})}
$$

where $\mathrm{LOD}_{0}$ is the length of a standard day $(86,400 \mathrm{~s})$.

\section{Results}

Our results show that Earth's spin changes induce an asymmetric response of VEI $\geq 3$ explosive eruptions along "W"- vs. "E"-directed subduction zones (Fig. 1). In fact, during the 1850-2009 time span, the ratio between the number of eruptions along E-directed and Wdirected subduction zones, $r_{\mathrm{E} / \mathrm{W}}$, is $166 / 256=0.65$. We introduce $\Delta \mathrm{LOD}_{\text {eff, }}$, defined as the difference $\Delta \mathrm{LOD}_{n}$ $\triangle \mathrm{LOD}_{n-1}$ where $n$ represents a year in the interval 1850 2009. An eruption occurred during the year $n$ has an associated $\Delta \mathrm{LOD}_{\text {eff }}=\Delta \mathrm{LOD}_{n-1}-\Delta \mathrm{LOD}_{n-2}$. This choice is based on the work by Palladino and Sottili (2014) showing how statistically significant anomalies of LOD appear 5 years before large explosive eruptions, with the highest anomalies recorded 1 or 2 years before each volcanic event. During the same time period, $\Delta \mathrm{LOD}_{\text {eff }}$ oscillate between +0.58 and $-0.76 \mathrm{~ms}$ with a central value of $0.00 \mathrm{~ms}$ (Fig. 2a). We note that, in the year after important Earth's spin decelerations (increasing LOD values above the 75th percentiles of the $\triangle \mathrm{LOD}_{\text {eff }}$ annual changes recorded since 1850), $r_{\mathrm{E} / \mathrm{W}}$ increases to 0.87 , with an increase of $\sim 35 \%$ (Fig. 2a). Conversely, an opposite trend is observed in the year after significant Earth's spin accelerations with $\Delta \mathrm{LOD}_{\text {eff }}$ below the 25 th percentile, when $r_{\mathrm{E} / \mathrm{W}}=0.46$ decreases by $\sim 28 \%$.

The statistical significance of the response of explosive eruptions on $\triangle \mathrm{LOD}_{\text {eff }}$ is tested and validated through a Monte Carlo technique. In other words, we evaluated quantitatively the probability that the observed correlation between LOD and volcanic eruptions may derive from a random pattern (null hypothesis). This statistical approach, widely applied in the scientific literature, was also recently used to evaluate quantitatively the correlations within short data records (Iles et al. 2013; Ting

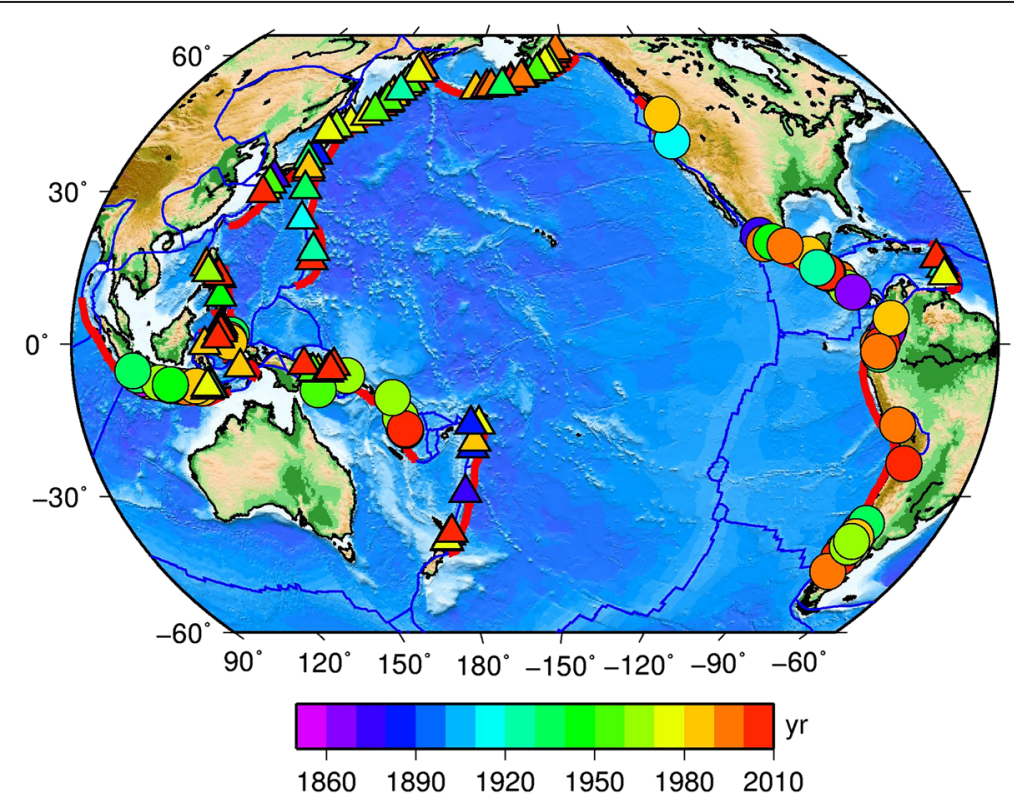

Fig. 1 Locations of the 422 explosive eruptions with VEI $\geq 3$ since AD 1850 to 2009, analysed along main subduction zones (red lines). Circles and triangles represent volcanoes located in $\mathrm{E}$ (or NE)- and W (or SW)-directed subduction zones, respectively. Colours are related to the year of the eruptions. Blue lines represent plate boundaries (DeMets et al. 2010). Map generated with the Generic Mapping Tools (the Generic Mapping Tools available online at http://www.soest.hawaii.edu/gmt/) 

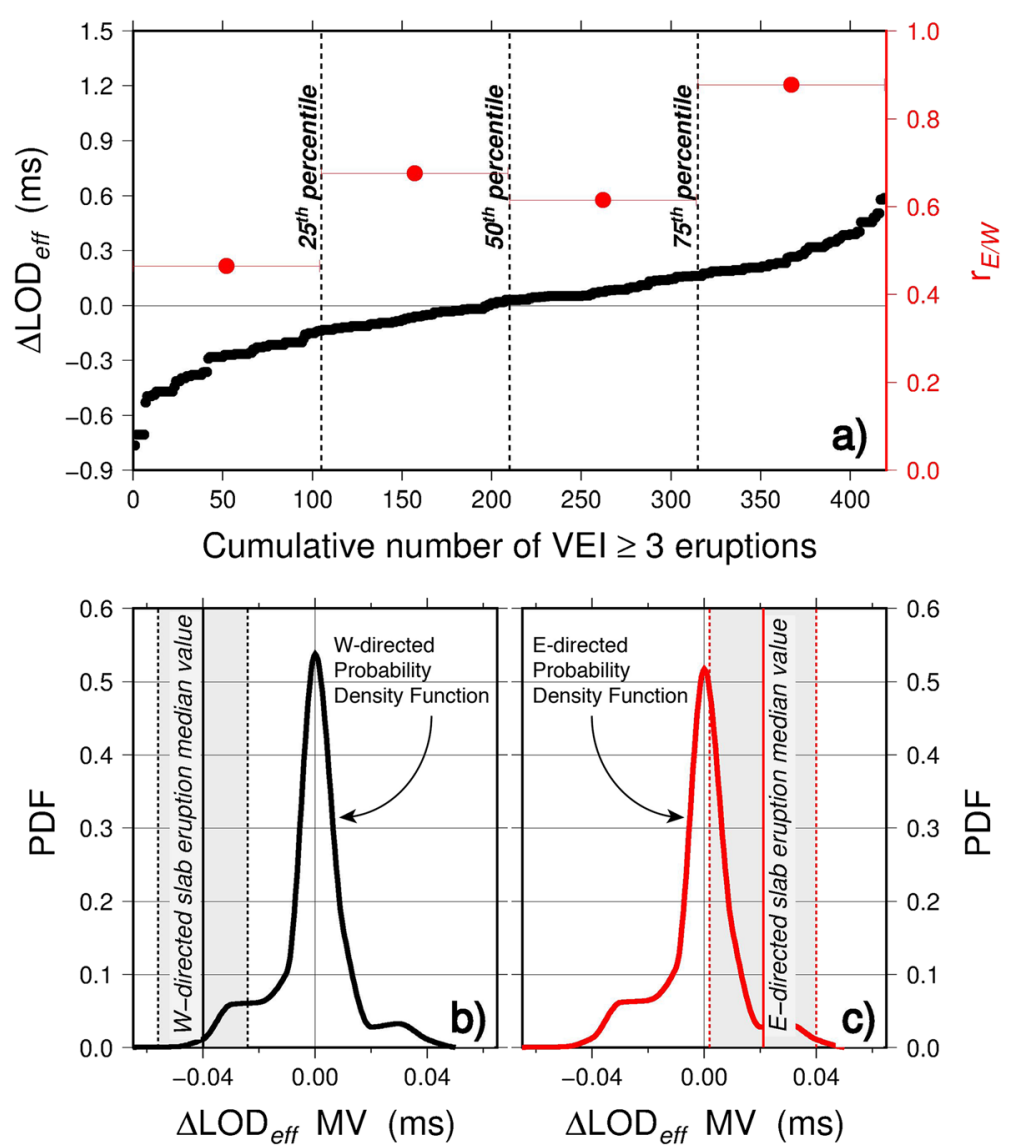

Fig. 2 a Cumulate curve of $\triangle L O D_{\text {eff }}$ prior to major explosive eruptions (422 events with $V E I \geq 3$ ) since 1850 and corresponding $r_{E / W}$ ratio, calculated

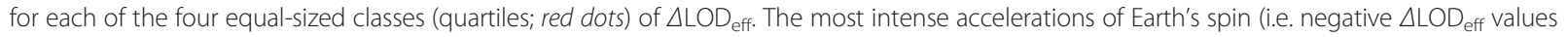
below the 25th percentile) are followed by an increase of the number of eruptions along W (or SW)-directed subduction zones and by a decrease of eruptions along the $\mathrm{E}$ (or NE)-directed (i.e. $r_{\mathrm{E} N \mathrm{~W}}$ ratio decreases by $28 \%$ below the mean value). For the definition of " $\mathrm{E}$ " and "W" subduction classes, see the text. After significant Earth's spin deceleration (i.e. $\Delta \mathrm{LOD}_{\text {eff }}$ increases above the 75 th percentile), $r_{E / W}$ ratio increases by $35 \%$ above the mean. Cumulative probability density function (PDF) of year-to-year LOD changes (median values) obtained from $10^{4}$ Monte Carlo simulations (b and $\mathbf{c}$ ). The two synthetic PDF curves refer to the distribution of $\triangle \mathrm{LOD}_{\text {eff }}$ changes (median values) for a sample of 256 events (i.e. corresponding to the number of eruptions that occurred on W-directed slabs) (b) and for a sample of 166 events (i.e. number of eruptions on E-directed slabs) (c) during the 1850 to 2009 time interval. Vertical dotted lines represent the mean standard deviation confidence levels. The comparison with the synthetic PDF distributions indicates that the observed LOD changes before eruptions on W-and E-directed slabs are significant at the $>98$ and $>95 \%$ confidence intervals, respectively

et al. 2011). Specifically, we consider Earth's spin changes preceding VEI $\geq 3$ events along E-directed subduction zones (i.e. $\Delta \mathrm{LOD}_{\text {eff, }}$ median value $=-$ $0.041 \mathrm{~ms}$ ) and those preceding VEI $\geq 3$ events along $\mathrm{W}$ - and or SW-directed subduction zones (i.e. $\Delta \mathrm{LO}-$ $D_{\text {eff }}$ median value $=0.021 \mathrm{~ms}$ ). From synthetic probability density functions (PDFs) obtained from $10^{4}$ Monte Carlo simulations, we evaluated the probability that the observed polarity in the year-to-year LOD changes may derive from a random sampling of the 1850-2009 LOD time series. This meant reshuffling the 160-year LOD time series like a deck of cards and sampling for $10^{4}$ times 166 (i.e. the number of VEI $\geq 3$ events along E-directed subduction zones) and 256 random years (i.e. the number of VEI $\geq 3$ along W-directed subduction zones) to obtain two synthetic PDF distributions of $\triangle \mathrm{LOD}_{\text {eff }}$ median values relative to $\mathrm{E}$ - and $\mathrm{W}$-directed subduction zones, respectively (Fig. 2b, c). The range of variability of PDF distributions, showing a clear unimodal distribution, provides the confidence intervals: only results beyond 5-95 \% from the Monte Carlo method were considered significant. The comparison with the synthetic PDF distributions (Fig. 2b, c) indicates that the observed $\triangle \mathrm{LOD}_{\text {eff }}$, before eruptions on W- and E-directed slabs, are significant at the $>98$ and $>95 \%$ confidence intervals, respectively (Fig. 2b). 


\section{Influence of LOD changes on subduction volcanoes}

To understand how Earth's spin changes may influence explosive activity at subduction volcanoes, we propose a conceptual model which takes into account the effects of LOD changes on the crustal stress state in subduction zones (Fig. 3). Previous works measured the effects of orbital and rotational variations on Earth's lithosphere local strain field changes (Milyukov et al. 2013) and on Jupiter and Saturn's icy satellites (Hoppa et al. 1999; Nimmo et al. 2007). Here, we focus on the capability of LOD-related stress changes to lead to the failure of the crustal rocks surrounding magma storage zones and thus to act as trigger mechanism for volcanic eruptions. In this regard, we use the term "trigger" to denote a process able to promote a fracturing event of magma chamber wall rocks on the condition that it acts in combination with the intrinsic dynamics of volcanoes (i.e. magma crystallization and differentiation, volatile pressure build-up). We propose that the rupture of magma chamber wall rocks occurs when the differential stress generated by LOD oscillation reaches a critical threshold (see Mohr's circles, Fig. 4). The state of stress in the crust determined by plate tectonics is modified due to rotation instabilities. In E-directed subduction zones, where volcanic arcs develop in compressive tectonic regime, Earth's spin decelerations (i.e. LOD increase) induce an increase of the horizontal stress $\left(\sigma_{1}\right)$ due to a lower tension in the crust and thus a larger differential stress favouring wall rock failure. On the contrary, in Wdirected subduction zones, where volcanism develops in back-arc extensional regime, Earth's spin accelerations (LOD decrease) produce larger differential stresses by enhancing the $\sigma_{3}$ tensor (Fig. 4).

To evaluate the presence of any contingent stress changes in the crust, related to Earth's spin variations, we propose a simple model able to support statistical results. Our intent here is not to solve the problem entirely. We evaluate whether the $\Delta \mathrm{LOD}_{\text {eff-related stress }}$ can be comparable with the other computed stress values, associated with other physical process (e.g. decompression rates related to glacio-eustatism or tidal forces), capable to promote the triggering of volcanic eruptions. We consider a rotating spherical shell with a specific thickness $d=R_{2}-R_{1}=20 \mathrm{~km}$, where $R_{2}=6371 \mathrm{~km}$ is the external radius, i.e. the Earth's radius, and $R_{1}$ is the internal radius (i.e. $R_{1}=6351 \mathrm{~km}$ ), subjected to centrifugal effects on its mass. At the equatorial plane, the spherical shell becomes a circular crown. Under this cylindrical approximation, we are going to evaluate the stress generated on this rotating thick ring, subjected to a radial pressure due to centrifugal effects, using equations derived by Hearn (1997).

Inertial forces do not affect the dynamics of the Earth's interior, and, with a constant Earth's rotation, the centrifugal force does not affect the gravitational-centrifugal force equilibrium on the Earth's surface. However, the rotational instability implies relatively small variations of

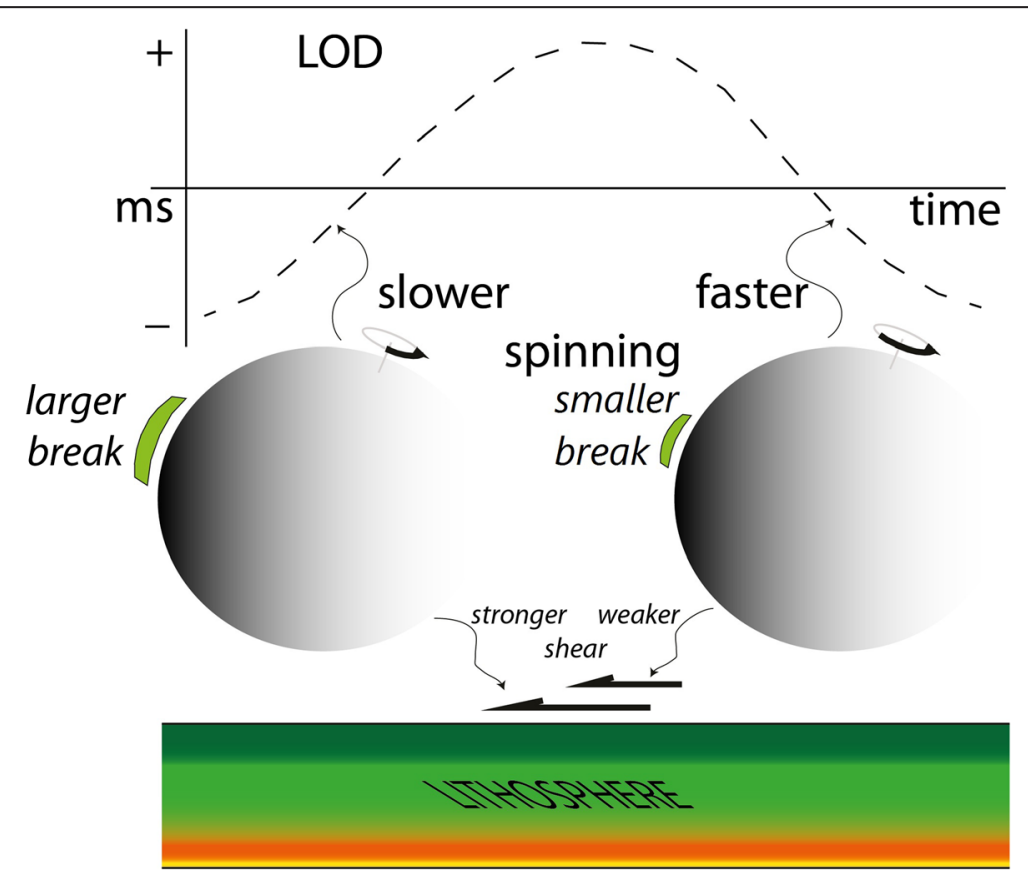

Fig. 3 Explanation of LOD-determined shear stress oscillation in the lithosphere. This should respectively determine an increase and a decrease of the shear stress acting on the lithosphere 


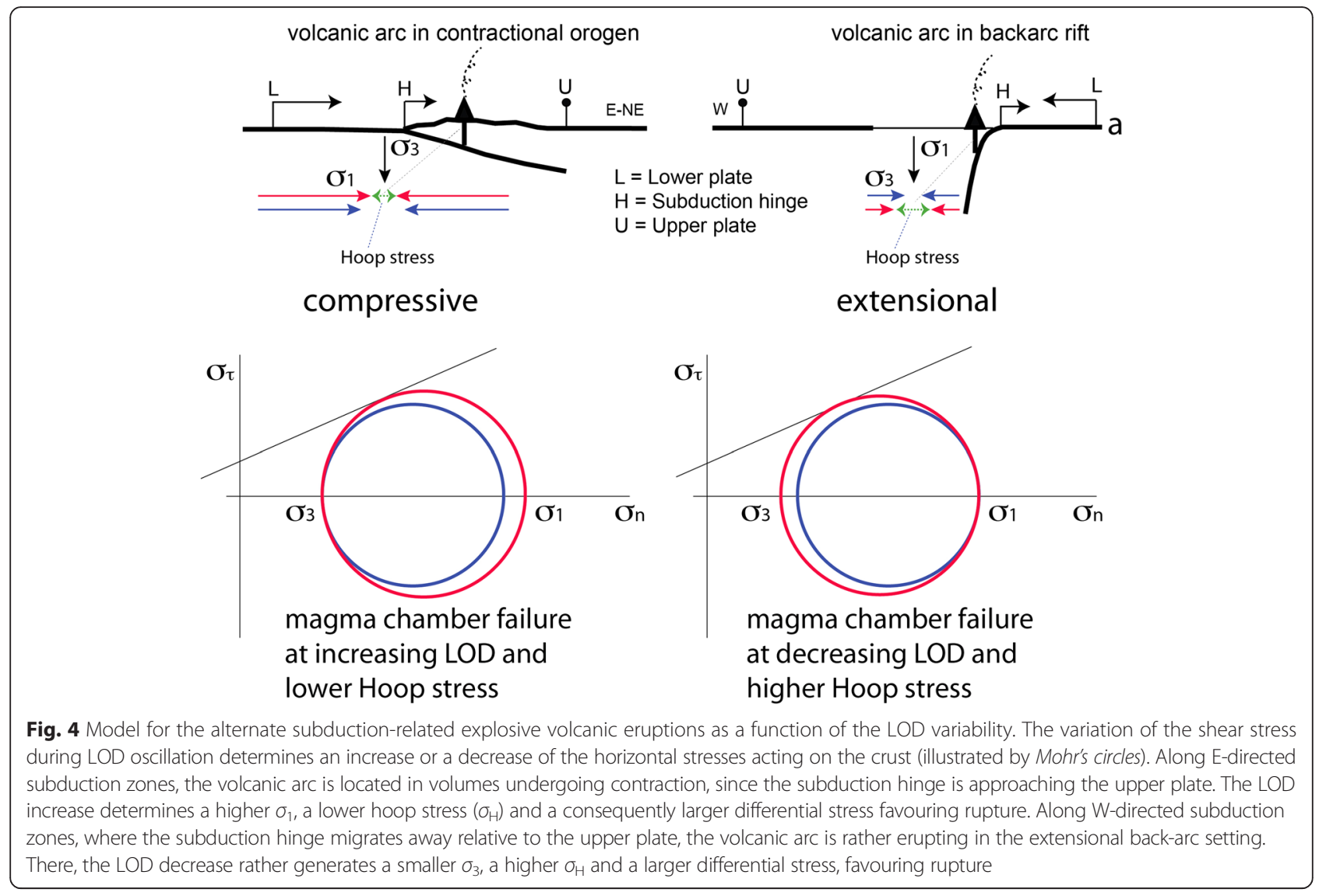

the centrifugal component, with a net effect on the balance between gravitational and centrifugal forces, providing a continuous, relatively small dynamic inconstancy. This yearly disequilibrium produces unsettled values of the tension applied on the thick ring (i.e. the crust), and, consequently, a variable circumferential stress, or hoop stress $\left(\sigma_{\mathrm{H}}\right)$, is generated (Hearn 1997).

Here, we compute the tensional hoop stress in the Earth crust, assuming, for sake of simplicity, a planar model on the equatorial plane. This corresponds to evaluate the radial $\sigma_{R}$ and hoop $\sigma_{\mathrm{H}}$ stresses of a rotating circular crown, or a thick ring, with a thickness of $d=$ $20 \mathrm{~km}$, as a function of the radial distance, $r$; the crust Poisson's ratio, $v$; the crust density, $\rho_{\mathrm{C}}$; the Earth angular velocity, $\omega$; and the internal and external circular crown radii, $R_{1}$ and $R_{2}$ (see Appendix). To analyse the variations of the radial and hoop stresses in our ideal crust that depend on $\triangle \mathrm{LOD}_{\text {eff }}$ changes, under the planar model approximation, we define here the effective radial $\sigma_{R}$ and hoop $\sigma_{\mathrm{H}}$ stresses as the $\sigma_{R}$ and $\sigma_{\mathrm{H}}$ obtained with Eqs. (2) and (3), derived by Hearn (1997), per unit of ms, respectively. Figure 5 shows the variations of the effective radial, $\sigma_{R}$, and hoop, $\sigma_{\mathrm{H}}$, stresses, as a function of $r$ in the crust. We notice that for this particular geometry approximation, the equations derived by Hearn (1997) for a thick ring, used here to compute radial and hoop stresses, result in quite different values, when considering our ideal crust at the equatorial plane. We obtain that the effective radial $\sigma_{R}$ is zero at the base and at the top of the crust $\left(R_{1}=6351\right.$ and $R_{2}=6371 \mathrm{~km}$, respectively), with a maximum values of $2.40 \mathrm{~Pa} / \mathrm{ms}$ in the middle crust (Fig. 5). On the contrary, we have higher values (i.e. five orders of magnitude) for the hoop stress in the whole ideal geometry, with a maximum value of $600 \mathrm{kPa} / \mathrm{ms}$ at the base of the crust (Fig. 5). We consider these results, obtained at the equator, as the maximum values that can be reached when modelling with the simple approximations of a thick ring geometry and an ideal crust. In fact, we may expect even different values for the hoop stress if modelling with a rotating spherical shell, knowing that the centrifugal potential is greatest at the equator (i.e. the hoop stress would decrease as a function of the latitude) and also taking into account the unelastic behaviour of the Earth, crust heterogeneities and interactions with the other spherical shells (e.g. the mantle).

In this perspective, variations of $\Delta \mathrm{LOD}_{\text {eff }}$ (1850 to 2009 period) and of the corresponding quantity $\Delta \omega_{\text {eff }}=$ $\omega_{n}-\omega_{n-1}$, being the difference of the Earth's rotation rate, over two consequent years are reported in 

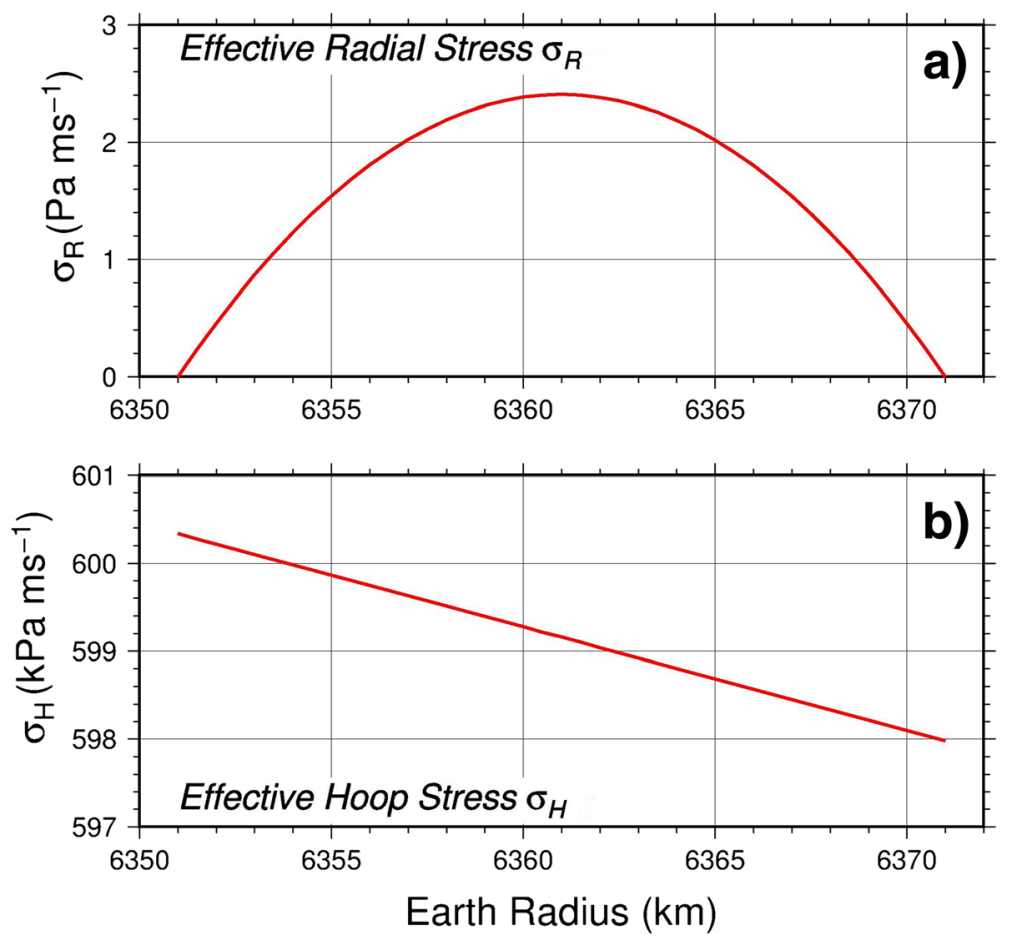

Fig. 5 Effective radial (a) and hoop (b) stresses in the Earth's crust. Theoretical computations for an ideal and homogeneous crust with both constant thickness $d=20 \mathrm{~km}$ and density $\rho_{\mathcal{C}}=2800 \mathrm{~kg} \mathrm{~m}^{-3}$ show that radial stress is almost negligible with respect to the hoop stress, being the maximum values reached of $2.40 \mathrm{~Pa}$ and $600 \mathrm{kPa}$, in the middle and at the base of the crust, respectively. Quantities are computed at the equatorial plane and reported per unit of milliseconds

Additional file 1: Figures S11 and S12, respectively. Thus, following the model by Hearn (1997), we obtain the variation of the hoop stress in the crust, $\Delta \sigma_{\text {Heff }}$ (Additional file 1: Figure S12), by Eq. (3), as a function

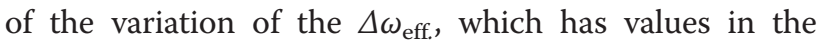
range between $\pm 400 \mathrm{kPa}$ for LOD variations of $1 \mathrm{~ms}$ (i.e. corresponding to $\pm 45 \mathrm{~Pa} / \mathrm{h}$ for typical annual $\Delta \mathrm{LOD}$ values). For comparison, decompression rates related to glacio-eustatism and tidal forces, considered among possible controlling factors of volcanic eruptions, range in the order of a few Pa per year (Kutterolf, et al. 2012) and $150 \mathrm{~Pa} / \mathrm{h}$ (Sottili et al. 2007), respectively, whilst magma volatile pressure build-up is reported around 400-720 Pa/h (Johnston and Mauk 1972; Jentzsch et al. 2001).

\section{Concluding remarks}

Most magma chambers are located within the shallow $20 \mathrm{~km}$ of the Earth's crust. The computation of the hoop stress in the crust, generated by the LOD annual variations, supports oscillations up to $0.4 \mathrm{MPa}$ of the horizontal stress acting on the wall rocks of magma chambers, which appears high enough to trigger rupture and promote magma ascent and eruption. Moreover, we show that VEI $\geq 3$ eruptions alternate along subduction zones characterized by either contractional or extensional regimes. Typically, contractional settings of subduction zones and related magmatic arc occur where the subduction hinge converges relative to the upper plate, whereas extensional settings are present where the subduction hinge diverges with respect to the upper plate (Fig. 3). Namely, these two opposed settings are frequently associated with E- or NE-directed subduction zones (plus Northern Japan) and W-directed slabs, respectively.

We conclude that differential stresses induced by Earth's spin variations appear quite relevant in magnitude as an additional factor of volcanic forcing, supporting the above statistical evidence for their clockwork role in eruptive activity in subduction geodynamic settings.

\section{Appendix}

Monte Carlo analysis

The quantitative evaluation of the probability that the observed correlation between LOD changes and volcanic eruptions may derive from a random pattern (null hypothesis) was performed through the Monte Carlo method. Specifically, we evaluated the probability that the observed polarity in the year-to-year LOD changes (median values) may derive from a random sampling of the 1850-2009 LOD time series. This meant reshuffling the 1850-2009 LOD time series like a deck of cards to obtain two synthetic probability density functions (PDFs); 
the first PDF refers to $10^{4}$ samples of 256 events (i.e. corresponding to the number of eruptions that occurred on W-directed slabs since 1850), and the second PDF refers to $10^{4}$ samples of 166 events (i.e. number of eruptions on E-directed slabs since 1850). The range of variability of PDF distributions provides the confidence intervals, and only results below $5 \%$ and above $95 \%$ from the Monte Carlo method were considered significant (Fig. 2).

\section{Hoop stress in the Earth crust model}

We assumed a planar model on the equatorial plane. Following Hearn (1997), the radial $\sigma_{R}$ and hoop $\sigma_{\mathrm{H}}$ stresses of a rotating circular crown, or a thick ring, with a thickness of $d=20 \mathrm{~km}$, are given by:

$$
\begin{aligned}
& \sigma_{R}(r)=(3+v) \frac{\rho_{\mathrm{C}} \omega^{2}}{8}\left[R_{1}^{2}+R_{2}^{2}-\frac{R_{1}^{2} R_{2}^{2}}{r^{2}}-r^{2}\right] \\
& \sigma_{\mathrm{H}}(r)=\frac{\rho_{\mathrm{C}} \omega^{2}}{8}\left[(3+v)\left(R_{1}^{2}+R_{2}^{2}+\frac{R_{1}^{2} R_{2}^{2}}{r^{2}}\right)-(1+3 v) r^{2}\right]
\end{aligned}
$$

where $r$ is the radial distance from the Earth's centre; $v$ is the crust Poisson's ratio; $\rho_{\mathrm{C}}$ is the crust density; $\omega$ is the Earth angular velocity; and $R_{1}$ and $R_{2}$ are the internal and external circular crown radii, i.e. here, $d=R_{2}-R_{1}$ equals the thickness of the crust. A full description of physical quantities and parameters is reported in Additional file 1: Table S2.

The effective radial $\sigma_{R}$ and hoop $\sigma_{\mathrm{H}}$ stresses, as the $\sigma_{R}$ and $\sigma_{\mathrm{H}}$ obtained with Eqs. (2) and (3), per unit of time (ms) are related to the variations of the radial and hoop stresses in the crust as a function of LOD changes.

\section{Additional file}

\section{Additional file 1: Supplementary information. Figures S1 to S9,} Locations of volcanic explosive eruptions with $V E I \geq 3$ along main subduction zones. Figure S10, Mean azimuth of the normal lines to the trench for the analysed subduction zones. Figure S11, Length of the day annual mean changes (LOD) in the 1840 to 2009 period. Figure S12, Yearly effective Earth's angular velocity variation and yearly effective hoop stress per unit of ms computed at the base of the crust. Table S1, Mean azimuth of the normal lines to the trench for the analysed subduction zones. Table S2, Physical quantities and parameters used for hoop stress computations. Table S3, List of the 422 explosive eruptions with VEI $\geq 3$ since AD 1850 to Present.

\section{Competing interests}

The authors declare that they have no competing interests.

\section{Authors' contributions}

GS and DMP proposed the ideas for this study. MC and CD contributed to develop the methods. GS and MC analysed the data and contributed the materials/analysis tools. All the authors wrote jointly the paper and discussed extensively the results and the interpretations. All authors read and approved the final manuscript.

\section{Acknowledgements}

We would like to thank the two anonymous reviewers and Prof. Yosuke Aoki who provided constructive comments. Discussions with Edie Miglio, Marco Ligi and Fabio Trippetta were very fruitful. This work was co-funded by PNRA 2013 grant 2013/B1.02.

\section{Author details}

${ }^{1}$ Istituto di Geologia Ambientale e Geoingegneria (IGAG)—CNR, 00015 Monterotondo, Rome, Italy. ${ }^{2}$ Dipartimento di Scienze della Terra, Sapienza-Università di Roma, P.le A. Moro 5, 00185 Rome, Italy. ${ }^{3}$ Istituto di Geologia Ambientale e Geoingegneria, CNR, Sez. Sapienza, c/o Dipartimento di Scienze della Terra, Sapienza-Università di Roma, P.le A. Moro 5, 00185 Rome, Italy.

Received: 22 June 2015 Accepted: 16 December 2015

Published online: 30 December 2015

\section{References}

Anderson DL (1974) Earthquakes and the rotation of the earth. Science 186:49-50 Barnes RTH, Hide R, White AA, Wilson CA (1983) Atmospheric angular momentum fluctuations, length-of-day changes and polar motion. Proc $R$ Soc Lond 387:31-73

Bizouard C, Gambis D (2009) The combined solution C04 for Earth Orientation Parameters, recent improvements, Springer Verlag series, Series International Association of Geodesy Symposia, Vol. 134, Hermann (Ed.), 330 p., ISBN: 9783-642-00859-7.

Chao BF, Chung W, Shih Z, Hsieh Y (2014) Earth's rotation variations: a wavelet analysis. Terra Nova 26:260-264

Crespi M, Cuffaro M, Doglioni C, Giannone F, Riguzzi F (2007) Space geodesy validation of the global lithospheric flow. Geophys J Int 168:491-506. doi:10. 1111/j.1365-246X.2006.03226.x

Cuffaro M, Doglioni C (2007) Global kinematics in the deep versus shallow hotspot reference frames. In: Foulger GR, Jurdy DM (eds) Plates, plumes, and planetary processes, Geol Soc Am Spec Pap., pp 359-374. doi:10.1130/2007. 2430(18)

DeMets C, Gordon RG, Argus DF (2010) Geologically current plate motions. Geophys J Int 181:1-80

Doglioni C, Carminati E, Cuffaro M, Scrocca D (2007) Subduction kinematics and dynamic constraints. Earth Sci Rev 83:125-175. doi:10.1016/j.earscirev.2007.04.001

Doglioni C, Ismail-Zadeh A, Panza G, Riguzzi F (2011) Lithosphere-asthenosphere viscosity contrast and decoupling. Phys Earth Planet Int 189:1-8

Doglioni C, Panza GF (2015) Polarized plate tectonics. Adv Geophys 56(3):1-167, http://dx.doi.org/10.1016/bs.agph.2014.12.001

Hayes GP, Wald DJ, Johnson RL (2012) Slab1.0: a three-dimensional model of global subduction zone geometries. J Geophys Res 117:B01302. doi:10.1029/ 2011JB008524

Hearn EJ (1997) Mechanics of materials vol 2, 3rd edn. Heinmann, Butter Worth Hoppa G, Randall Tufts B, Greenberg R, Geissler P (1999) Strike-slip faults on Europa: global shear patterns driven by tidal stress. Icarus 141:287-298

Ide S, Tanaka Y (2014) Controls on plate motion by oscillating tidal stress: evidence from deep tremors in western Japan. Geophys Res Lett 41:3842-3850. doi:10.1002/2014GL060035

Iles CE, Hegerl GC, Schurer AP, Zhang Z (2013) The effect of volcanic eruptions on global precipitation, J Geophys Res Atmos, 118, doi:10.1002/jgrd.50678.

Jault D, Gire C, Le Mouel JJ (1988) Westward drift, core motions and exchanges of angular momentum between core and mantle. Nature 333:353-356

Jentzsch G, Haase O, Kroner C, Winter U (2001) Mayon volcano, Philippines: some insight into stress balance. J Volcanol Geoth Res 109:205-217

Johnston MJS, Mauk FJ (1972) Earth tides and the triggering of eruptions from Mt. Stromboli, Italy. Nature 239:266-267

Kane RP, Trivedi NB (1990) Decade fluctuations in the rotation rate of the Earth in the last 200 years. Pageoph 132:771-799

Kutterolf S et al (2012) A detection of Milankovitch frequencies in global volcanic activity. Geology G33419:1, dx.doi.org/10.1130/G33419.1

Lambeck K (1980) The Earth's variable rotation: geophysical causes and consequences. Cambridge University Press, Cambridge, 450 pp

Le Mouël J-L, Blanter E, Shnirman M, Courtillot V (2010) Solar forcing of the semi-annual variation of length-of-day. Geophys Res Lett 37:L15307. doi:10. 1029/2010GL043185

Marcus SL, Chao Y, Dickey JO, Gegout P (1998) Detection and modeling of nontidal oceanic effects on Earth's rotation rate. Science 281:1656-1659 
Milyukov V, Mironov A, Kravchuk V, Amoruso A, Crescentini L (2013) Global deformations of the Eurasian plate and variations of the Earth rotation rate. J Geodyn 67:97-105, http://dx.doi.org/10.1016/j.jog.2012.05.009

Mound JE, Buffett BA (2003) Interannual oscillations in length of day: implications for the structure of the mantle and core. J Geophys Res 108:no. 2334. doi:10. 1029/2002JB002054

Nimmo F, Spencer JR, Pappalardo RT, Mullen ME (2007) Shear heating as the origin of the plumes and heat flux on Enceladus. Nature 447:289-291

Palladino DM, Sottili G (2014) Earth's spin and volcanic eruptions: evidence for mutual cause-and-effect interactions?. Terra Nova 26:78-84

Press F, Briggs P (1975) Chandler wobble, earthquakes, rotation, and geomagnetic changes. Nature 256:270-273

Riguzzi F, Panza G, Varga P, Doglioni C (2010) Can Earth's rotation and tidal despinning drive plate tectonics? Tectonophysics 484:60-73

Rosen RD, Salstein DA (1985) Contribution of stratospheric winds to annual and semiannual fluctuations in atmospheric angular momentum and the length of day. J Geophys Res 90(D5):8033-8041

Siebert L, Simkin T (2002) Volcanoes of the world: an illustrated catalog of Holocene volcanoes and their eruptions. Smithsonian Institution, Global Volcanism Program, Digital Information Series, GVP-3; www.volcano.si.edu

Sottili G (2014) Constraints on climate forcing by sulphate aerosols from seasonal changes in Earth's spin. Geophys J Int 197(3):1382-1386. doi:10.1093/gji/ggu099

Sottili G, Martino S, Palladino DM, Paciello A et al (2007) Effects of tidal stresses on volcanic activity at Mount Etna, Italy. Geophys Res Lett 34:L01311

Ting M, Kushnir Y, Seager R, Li C (2011) Robust features of Atlantic multidecadal variability and its climate impacts. Geophys Res Lett 38:L17705. doi:10.1029/ 2011GL048712

Varga P, Gambis D, Bus Z, Bizouard C (2005) The relation between the global seismicity and the rotation of the Earth, Observatoire de Paris, Systémes de reference tempsespace UMR8630/CNRS, 115-121.

Wang Q-L, Chen Y-T, Cui D-X, Wang W-P, Liang W-F (2000) Decadal correlation between crustal deformation and variation in length of day of the Earth. Earth Planets Space 52:989-992

\section{Submit your manuscript to a SpringerOpen ${ }^{\circ}$ journal and benefit from:}

- Convenient online submission

- Rigorous peer review

- Immediate publication on acceptance

- Open access: articles freely available online

- High visibility within the field

- Retaining the copyright to your article

Submit your next manuscript at $\gg$ springeropen.com 